\title{
OBITUARIO
}

\section{Doctor Alberto Carvacho Bravo: Del campo al mar, desde la agronomía a la carcinología}

\author{
Pablo Marcos Espinoza-Concha ${ }^{1,2}$ \\ ${ }^{1}$ Departamento de Español, Facultad de Educación y Humanidades, Universidad de Tarapacá, Casilla 6-D, Arica, Chile. \\ pespinozac@uta.cl \\ ${ }^{2}$ Programa de Doctorado en Comunicación Social, Facultad de Ciencias Sociales y de la Comunicación, Universidad del \\ País Vasco / Euskal Herriko Unibertsitatea
}

El tiempo hace bien su tarea: ¡Olvidamos! Con Alberto Carvacho Bravo, ingeniero agrónomo por la Uiversidad de Concepción, Chile, y doctor en Ciencias Naturales por la Universidad de Pierre et Marie Curie (Paris VI), Francia, ocurrió lo propio.

Supe de su fallecimiento el lunes 9 de abril de 2018 mientras revisaba bibliografía en la biblioteca del campus Leioa de la Universidad del País Vasco / Euskal Herriko Unibertsitatea, en Bilbao. ¡Qué paradoja! Trabajo sobre «memoria»; en particular, los naturalistas olvidados de Chile, los mismos que don Alberto quiso, admiró y respetó desde su niñez. Estos personajes, a sus cinco años de edad, le marcaron a fuego su camino a seguir; luego, de adulto, lo llevaron a desarrollar una vida profesional prolífica tanto en la academia como en la investigación científica, en Chile y el extranjero.

En su nombre no se publicaron obituarios en diarios ni revistas especializadas, tampoco en los órganos de difusión de universidades y corporaciones científicas a las que se vinculó. A Angol de Los Confines, su ciudad natal, no llegó la noticia. ¡Nada! ¡Olvidamos pronto! ¡Somos ingratos! Pero las reglas tienen excepciones: «Este miércoles 28 de junio de 2017 es un día triste para nuestro museo, pues se produjo el fallecimiento de Alberto Carvacho Bravo, director del Museo Nacional de Historia Natural entre los años 1996 y 1999», sostuvo la web institucional. También fue profesor e investigador de la Escuela de Agronomía de la Universidad de Chile, que lo premió como 'académico destacado' en 2002, el pie de la fotografía de la 'Casa de Bello' dice: «Junto al Rector de izquierda a derecha; Prof. Sonia Acevedo, Prof. Alberto Carvacho, Prof. Guillermo García, Prof. Bruno Razeto».

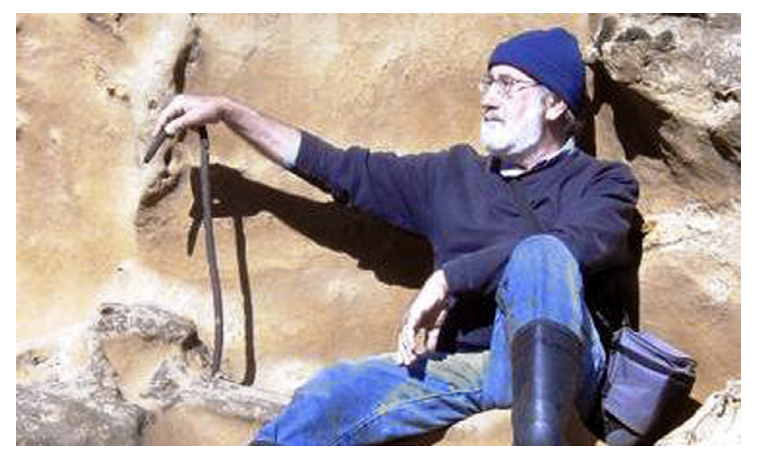

Doctor Alberto Carvacho Bravo en Ahuenco, Chiloé, Región de Los Lagos, Chile. Fotografía gentileza de Cristian Sepúlveda / Doctor Alberto Carvacho Bravo in Ahuenco, Chiloé, Los Lagos Region, Chile. Photograph courtesy of Cristian Sepúlveda 
La desaparecida Patricia Verdugo Aguirre, Premio Nacional de Periodismo 1997, escribió sobre él, en 1998: «Experto en Biogeografía y Carcinología, conocedor de la vida y milagros de camarones, isópodos, jaibas y paguros, el doctor Carvacho dejó Francia el 77 luego de permanecer más de seis años en el Museo, en la Station Biologique de Roscoff e incluso en las Antillas Francesas. Su huella continúa como investigador de la Escuela Superior de Ciencias Marinas, de la Universidad Autónoma de Baja California (Ensenada, México) y luego del Centro de Investigación Científica y Educación Superior de Ensenada (CICESE). México lo distinguió el año 85 otorgándole el grado de 'Investigador Nacional' grado dos (CONACYT), máximo nivel para un extranjero».

En aquella entrevista Alberto Carvacho Bravo reconoció como los maestros que marcaron su vocación a don «Nibaldo Bahamonde Navarro, Premio Nacional de Ciencias 1996 y al 'gringo' Dillman Bullock (que lo conectó con el mundo animal de su infancia angolina) hasta el profesor Forest en el Museo de Historia Natural de Paris, pasando por Patricio Sánchez y Luis Izquierdo cuando trabajó en la Escuela de Medicina de la Universidad Católica (63-67) y siguiendo con Humberto Maturana y Francesco di Castri en la Escuela de Ciencias de la Universidad de Chile (6873)». Asimismo, que su retorno a Chile fue en 1989, tras haber trabajado en el Instituto Oceanográfico de La Habana, que se desempeñó como académico y vicerrector del Instituto Profesional de Osorno, hoy Universidad de Los Lagos. Además, de ser autor de una cincuentena de artículos científicos publicados en revistas de corriente principal de alto impacto y de ponencias en congresos internacionales de su especialidad.

Testigo del cariño que le tuvieron sus estudiantes es la especie Fabia carvachoi, que le fue dedicada. En 1996, publicó en esta revista -que lo recuerda y lo despide- el artículo Nuevo registro de Metabetaeus minutus (Whitelegge) (Crustacea, Decapoda, Alpheidae) en Isla de Pascua.

-Buenas tardes. Buscamos a don Alberto Carvacho Bravo -dijimos.

-Don Alberto está ocupado, tiene reuniones toda la tarde, es más, toda la semana. No los podrá recibir -enfatizó la secretaria de la dirección del Museo Nacional de Historia Natural, Chile, ese enero de 1998.

-¡Por favor señora!, dígale que venimos desde Angol a hablar de Bullock, eso nada más. ¡Angol y Bullock! afirmamos con cierto temor y ya casi derrotados.

Nuestra insistencia con características de ruego cumplió su objetivo. Apareció raudo, vestía pantalón gris, chaleco rojo sin mangas y una chaqueta azul; de barba y bigote blancos; a nuestro entender, sus lentes metálicos con vidrios gruesos eran los signos característicos de un investigador importante, reconocido por la comunidad científica internacional, de esos que actúan en las ligas mayores. De los que no conocíamos, pero que gracias a su ayuda y generosidad luego pudimos entrevistar, entre ellos, el profesor Nibaldo Bahamode Navarro, Premio Nacional de Ciencias 1996, y el ingeniero civil hidráulico y arqueólogo Hans Niemeyer Fernández, exdirector del Museo Nacional de Historia Natural (1921-2005).

-¡Pasen, pasen, adelante, adelante! -sentenció algo acelerado. La secretaria cumplió con su trabajo, le recordó al director los compromisos que él había contraído con antelación.

-¡Usted arregle eso!, hoy conversaré con mis amigos que vienen de Angol-le afirmó don Alberto. ¡Y no nos conocía!

-¡Por favor!, nos puede traer unos cafecitos -le solicitó con aire de súplica a su secretaria, cual niño que asume la autoría de alguna travesura.

Ese enero de 1998, Alberto Carvacho Bravo tenía 62 años, luego de escuchar la presentación que realizamos junto al ahora periodista Patricio Álvarez Navarro, nos dijo con energía: 'Bullock fue una puerta abierta a todo lo que fui después. El camino donde llegué, la dirección del Museo Nacional de Historia Natural, lo comencé a recorrer en el taller de Bullock cuando yo tenía cinco años, en El Vergel, Angol. Ponía mis manos en la mesa del comedor de su casa y me empinaba para ver todo lo que él tenía, en especial, cosas de los mapuche. ¡Ahí descubrí el mundo! 'iQué ganas de ser como Bullock cuando sea viejo!', me decía. Tendría que haber nacido en el exterior, con el talento de él y todo lo que tuvo Bullock. Yo creo que mi historia comenzó con Bullock, ¡sin duda! ¡Sin Bullock estaría haciendo otra cosa!’. 
Para nosotros fue la mejor respuesta, lo que queríamos escuchar. El personaje seleccionado para trabajar la tesis de grado: Dillman Samuel Bullock Lytle (Elba, Michigan, Estados Unidos, noviembre 28 de 1878 - Angol, Malleco, Chile, abril 05 de 1971) era reconocido en el concierto agronómico chileno, en particular, y científico, en general. Un NATURALISTA. Pero el tiempo había hecho su tarea, lo tenía olvidado para las futuras generaciones, hasta para algunos habitantes de Angol donde una avenida principal y un museo llevan su nombre. ${ }_{j} E l$ problema de la memoria!

Además, Alberto Carvacho Bravo conocía muy bien a 'Bullock-naturalista', lo quería, lo respetaba y lo reconocía como uno de sus maestros. También demostró su generosidad al nombrarnos una cantidad de científicos que podrían aportarnos en nuestra labor, fue una fuente directa; por último, escribió de su puño y letra una carta presentándonos a sus amigos investigadores. Sin dudas, un apoyo fundamental para que finalizáramos con éxito la tarea académica y nos tituláramos. Su nombre nos abrió puertas, nos allanó el camino.

«Si vienen al museo mañana traeré un regalo que me hizo Bullock, unas mariposas en una caja que él confeccionaba, con algodón, vidrio y papel negro. Me escribió una dedicatoria», nos aseguró. Para nosotros, ¡mucho mejor!, tendríamos otra jornada de conversación con don Alberto, con el hijo de doña Remedios Bravo viuda de Carvacho, la recordada directora de la Escuela Normal de Angol, a quien un monolito honra su memoria en los jardines del ex establecimiento, hoy Liceo 'Enrique Ballacey Cottereau' de la ciudad de Los Confines.

Hoy, a más de un año de su partida física, recuerdo con alegría las reuniones que sostuve con mi coterráneo Alberto Carvacho Bravo (Angol, Malleco, Región de La Araucanía, agosto 4 de 1935 - Contuy, Queilen, Región de Los Lagos, junio 28 de 2017). Saludo con gratitud su brillantez profesional, su amplia cultura y su tremenda humildad, la que le permitió reconocer cualidades en 'los otros' y decirlo públicamente. A modo de ejemplo, en la entrevista que le concedió a Patricia Verdugo dijo: «Su primer recuerdo del museo data de 1940 cuando su padre lo traía desde Angol en visita a la capital: Era 'el' Museo, a secas, y tengo grabada la imagen de una inconmensurable cantidad de pajaritos embalsamados. Su segundo contacto lo hizo siendo estudiante de Agronomía y ayudante de Zoología, cuando le encargaron desempolvar la colección de animales del eminente sabio Carlos Porter. Fui recibido en el laboratorio del mejor, Nibaldo Bahamonde, un chilote que tiene un solo defecto gigantesco: su inaudita modestia».

También me congratulo por la suerte que tuve al conocerlo. Hoy, a través de este obituario/homenaje -aunque atrasadole agradezco su generosidad, por compartirme parte de sus anécdotas y su visión de mundo. Un mundo que para él creció al recorrerlo y, lo mejor, donde lo habitó dejó su impronta de hombre de bien, laborioso y honesto; dedicado a la educación, al desarrollo de la agronomía, de la carcinología y de las ciencias naturales. Lo anterior quedó plasmado en las entrevistas que nos concedió hace veinte años en la oficina que ocupaba como Director del Museo Nacional de Historia Natural.

Si bien en aquella oportunidad el punto central fue Dillman Bullock; hoy, a través de los dichos hacia su primer mentor y con la distancia que dan cuatro lustros, Alberto Carvacho Bravo nos enseñó sobre los naturalistas, las ciencias naturales, la genética, la especialización científica y, también, nos dejó claro su infinito amor por la naturaleza, en general. Habló de temas profundos, contingentes y relevantes que solamente una persona privilegiada y con un intelecto superior puede vislumbrar, entender y explicar con simpleza.

-No sé cuándo conocí a Bullock, él me empezó a ver primero. Mis recuerdos se me pierden en el cuándo. Lo que sí es cierto, es que mi relación pasó por la que tenía mi madre con él, ella era directora de la Escuela Normal de Angol y él, director de la Escuela Agrícola El Vergel, mantenían un vínculo entre directores. Estos establecimientos eran dos centros importantes en la región y ellos, como docentes, se sentaban a conversar. Además, yo era compañero de Joyce Reed, hija del administrador del Fundo El Vergel.

(...) Lo que más me impresionó -y hasta ahora- fue su taller o laboratorio que tenía en su casa. Esa casa tan norteamericana, con doble puerta, una con 'mosquetera' y la otra normal, como en las películas del oeste. Tenía unas mesas enormes que, con mi edad y mi porte, con mi nariz llegaba al borde y miraba para adentro; no sé, tendría cuatro o 
cinco años, pero allí había de todo. De todo quiere decir: insectos que estaban siendo estirados, animales en formalina o alcohol, muchos restos de cántaros, urnas funerarias en proceso de parchado, piedras horadadas, plantas herborizándose, herbarios en vías de montaje y una cantidad de todo lo que significa naturaleza. Asimismo, el hombre metido en ella, y había que sumarle la antigua cultura mapuche de la zona.

Para un chiquillo chico, como yo, era descubrir el mundo a través de ese personaje que no sabía de dónde había llegado, no sé de qué galaxia y me mostraba todo. Todo lo que pudiera preguntarle a todos los seres humanos del mundo, todo me lo entregaba Bullock... ¡Me contaba de todo y con él sabía de todo! No tenía más que mi pueblo, al que quería y era lo mejor del mundo, entonces, mi mundo se reducía a eso. Un chiquillo chico viviendo en un pueblo pequeño, fantástico y maravilloso, donde podía preguntarle a muchas personas para conocer el mundo o bien recurrir a una sola, Bullock. Todo lo que me interesaba me lo contaba él. Además, me lo decía con una voz tan pintoresca. Hasta la última vez que lo vi, como en 1950, hablaba como Tarzán, nunca aprendió bien el español.

Su frase famosa: «Los pajaritos andaron, andaron, andaron», cuando se refería a las migraciones. Hasta hoy veo al 'gringo' decir, «andaron, andaron, andaron». Eso me hizo pensar de niño que yo quería ser 'naturalista'. Después descubrí que era una palabra del siglo pasado y que hoy no existen los naturalistas. Que había que ser zoólogo y, dentro de la zoología, ser entomólogo, malacólogo o carcinólogo, por ejemplo. Hoy no se puede ser naturalista. Mi ejemplo, soy ingeniero agrónomo y terminé trabajando en carcinología e hice un doctorado en crustáceos, es tan fino como eso.

(...) Él me mostró cosas tan extravagantes como la genética. Por ejemplo, los copihues (Lapageria rosea) y entender qué pasaba cuando yo veía en el campo copihues rojos, rojos, rojos y, de repente, uno blanco. Además, en El Vergel había copihues gigantescos y de todos los colores: Rojo, blanco, marfil, medios verdosos; de por qué y de dónde salían, esas cosas me las explicó Bullock. Me decía que ellos cruzaban plantas, que las elegían y luego hacían cosas de genética.

Es probable que en Chile, en ese tiempo, hubiera tres o cuatro personas dedicadas a la ciencia. Era la época de don Carlos Porter y aunque Bullock tenía una tremenda modestia, seguramente, era más sabio que éste. Porter se había educado en Chile y reconozcamos que formar un científico -entonces y ahora- siempre será mejor en los Estados Unidos que acá. Entre un doctorado en Estados Unidos y un sabio Porter, que debe haber sido profesor, una profesión terriblemente meritoria, hay bastante diferencia. Hay que dejar en claro que para ser científico hay que tener una muy buena formación y Bullock la tenía. Es mejor tener un doctorado en zoología para dedicarse a la ciencia que ser profesor de biología. A la inversa, para hacer clases es mejor el profesor de biología. ¡Cada uno en su lugar!

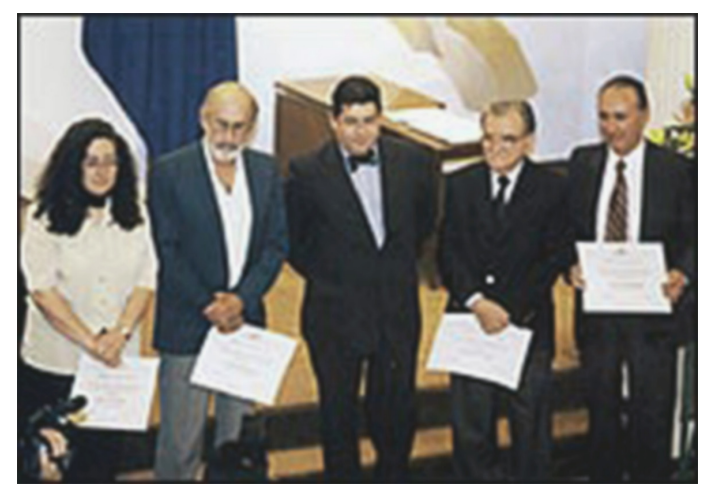

Ceremonia de entrega de la distinción a los mejores docentes de pregrado de la Facultad de Ciencias de la Universidad de Chile, diciembre de 2012. De izquierda a derecha: Prof. Sonia Acevedo, Prof. Alberto Carvacho, Prof. Luis Riveros (Rector de la Universidad de Chile), Prof. Guillermo García, Prof. Bruno Razeto. Fotografía gentileza Universidad de Chile / Award ceremony for the Best Undergraduate Teachers of the Facultad de Ciencias of the Universidad de Chile, December 2012. From left to right: Prof. Sonia Acevedo, Prof. Alberto Carvacho, Prof. Luis Riveros (Rector of the Universidad de Chile), Prof. Guillermo García, Prof. Bruno Razeto. Photograph courtesy of Universidad de Chile 
Bullock tuvo un mérito muy grande y aquí sobrepasa a casi todos los naturalistas. Él llegó al nivel que llegó y es apreciado en las ciencias chilenas hoy día no habiéndose movido casi nunca de Angol. Si uno mira dónde estuvieron los científicos de la época, jen Santiago! Los que hemos sido provincianos y por aquella fatalidad de la historia terminamos en la capital nos damos cuenta qué distinto es estar aquí; las posibilidades que ofrecía y las que había en Angol. Si quiero aprender Ciencias Naturales en Santiago puedo venir al Museo Nacional de Historia Natural. Bullock quiso hacer lo mismo, pero tuvo que crear un Museo de Historia Natural allá. Realmente, si uno lo piensa así, su obra es titánica. ¿Qué hubiera sido de Bullock en Santiago, por ejemplo, como director de este museo? 'Sepa Moya', a lo mejor un desastre. Tal vez, su vocación fue ser pionero.

Es buena la palabra pionero. Creo que ninguno de los que llegó a Santiago fue pionero; tal vez, Claudio Gay. Los demás llegaron a este edificio fantástico que ya tenía laboratorios. Es verdad que venían muertos de hambre del campo, es comprensible, estaban en el siglo pasado, pero había algo aquí en Santiago, siempre hubo algo, era la capital. Además, este museo se creó en 1830 y Claudio Gay empezó a hacer cosas. Bullock llegó a comienzo de este siglo a Angol y no había nada, tuvo que hacerlo todo. Todo su aporte lo hizo desde Angol y eso es lo importante.

(...) Tengo el último recuerdo de Bullock cuando yo era grande, un estudiante de agronomía, pero quería ser naturalista para dedicarme a los animales. Terminé la carrera, porque me sacaba buenas notas, además, un título hace falta, un cartón para cualquier cosa. Cuando iba a Angol lo pasaba a saludar. Una vez, en una de mis últimas visitas, me regaló una de esas cajitas que él mismo fabricaba -con algodón por detrás y un vidrio por delante- venían dos mariposas brasileñas, azules, brillantes, con alas bien fuertes, una grande arriba y una chica abajo. Atrás la dedicatoria dice: «Un cariñoso recuerdo del Museo Dillman Bullock». Siempre he tenido ese obsequio de Bullock en el escritorio de mi casa.

El doctor Alberto Carvacho Bravo, el carcinólogo provinciano que quiso ser naturalista, descansa en paz junto al mar en Chiloé, donde habitó sus últimos años. Su recuerdo de hombre de bien es iluminado por un contingente amplio de luces, el mismo que lo acompañó y adornó durante toda su vida.

\section{REFERENCIAS}

Álvarez-Navarro P \& P Espinoza-Concha. 1998. Dillman.S Bullock: Un naturalista, su vida como un ejemplo del saber multidisciplinario». Tesis de Periodista, Escuela de Periodismo, Universidad Austral de Chile, Valdivia, 498 pp.

Álvarez P \& P Espinoza. 2001. Dillman S. Bullock: El naturalista de La Araucanía, 222 pp. Ediciones Universidad Austral de Chile, Valdivia.

Museo Nacional de Historia Natural. 2009. Biobibliografía, 1980-2008. Publicación Ocasional del Museo Nacional de Historia Natural, Chile 61: 1-130. [p. 29]

Museo Nacional de Historia Natural. 2017. Ex Director del MNHN: En memoria de Alberto Carvacho Bravo (1935-2017). $<\mathrm{http}: / /$ www.mnhn.cl/sitio/Contenido/Noticias/77314:En-memoria-de-Alberto-Carvacho-Bravo-1935-2017>

Saavedra M, A Carvacho \& S Letelier. 1996. Nuevo registro de Metabetaeus minutus (Whitelegge) (Crustacea, Decapoda, Alpheidae) en Isla de Pascua. Revista de Biología Marina 31(2): 117-122.

Verdugo P. 1996. Doctor Alberto Carvacho, nuevo director del Museo Nacional de Historia Natural de Historia Natural. Noticiario Mensual del Museo Nacional de Historia Natural, Chile 326: 20-22. 